\title{
Interplay between multiple length and time scales in complex chemical systems*
}

\author{
BIMAN BAGCHI ${ }^{1}$ and CHARUSITA CHAKRAVARTY ${ }^{2} * *$ \\ ${ }^{1}$ Solid State and Structural Chemistry Unit, Indian Institute of Science, Bangalore 560012 \\ ${ }^{2}$ Department of Chemistry, Indian Institute of Technology, New Delhi 110016 \\ e-mail: charusita@gmail.com
}

\begin{abstract}
Processes in complex chemical systems, such as macromolecules, electrolytes, interfaces, micelles and enzymes, can span several orders of magnitude in length and time scales. The length and time scales of processes occurring over this broad time and space window are frequently coupled to give rise to the control necessary to ensure specificity and the uniqueness of the chemical phenomena. A combination of experimental, theoretical and computational techniques that can address a multiplicity of length and time scales is required in order to understand and predict structure and dynamics in such complex systems. This review highlights recent experimental developments that allow one to probe structure and dynamics at increasingly smaller length and time scales. The key theoretical approaches and computational strategies for integrating information across time-scales are discussed. The application of these ideas to understand phenomena in various areas, ranging from materials science to biology, is illustrated in the context of current developments in the areas of liquids and solvation, protein folding and aggregation and phase transitions, nucleation and self-assembly.
\end{abstract}

Keywords. Multiple time-scale methods; ultrafast spectroscopy; has ingle-molecule spectroscopy; energy landscapes; protein folding; protein aggregation; solvation; water; hydration; hydrophobic effect; nucleation; nanoscale self-assembly.

\section{Introduction}

An understanding of the diverse range of structures and dynamical processes seen in chemical systems is necessary in order to comprehend many phenomena, in physics, chemistry and biology. Chemical diversity is generated by the interaction of well-defined components, electrons and nuclei, whose behaviour is governed by well-understood forces and equations of motion, corresponding to Coulombic forces and classical or quantum mechanics. Despite a century of progress in experimental, theoretical and computational physical chemistry, the application of physical principles to understand many aspects of such systems with reasonable predictive accuracy still poses a challenge. A major cause of difficulty is that the behaviour of a molecular system is determined by processes operating on a multiplicity of length and time scales. Electrons moving on time scales of $10^{-18} \mathrm{~s}$ (attoseconds) determine the interatomic

\footnotetext{
*Reproduced from 'Current Trends in Science' Platinum Jubilee Special Publication, Indian Academy of Sciences, Bangalore, 2009, pp. 67-78 with minor editorial changes. **For correspondence.
}

interactions. Atomic motions are typically associated with length and time scales of $10^{-10} \mathrm{~m}$ and $10^{-15} \mathrm{~s}$, respectively; for example, time periods of molecular vibrations and rotations are of the order of $10^{-13} \mathrm{~s}$ and $10^{-12} \mathrm{~s}$, respectively. Many important chemical reactions, like electron and proton transfer, occur on timescales of $10^{-9}$ to $10^{-12} \mathrm{~s}$. The mesoscopic level of organization associated with many soft matter systems, such as colloids, proteins and micelles, is associated with length/time scales of $10^{-6} \mathrm{~m} / 10^{-9} \mathrm{~s}$. Enzyme kinetics usually proceed with time constants of a few milliseconds, although the actual bond breaking/forming may occur in a few nanoseconds.

Tailoring the functional properties of materials on a macroscopic scale $(1 \mathrm{~m} / 1 \mathrm{~s})$ involves averaging over scales smaller by several orders of magnitude in time and space. While statistical mechanics does provide the theoretical framework for such an averaging process, the actual implementation is complex because distinct experimental and computational approaches are typically required to understand the behaviour of materials at different levels of the hierarchy. For example, ab initio computational methods for electronic structure focus on obtaining the elec- 
tronic energies and wave functions for a given atomic arrangement. ${ }^{1,2}$ In the macroscopic limit, finite element approaches, based on continuum approximations that completely ignore atomistic detail, are often used to model functional properties of materials, such as response to mechanical stress.

One of the traditional concerns of physical and theoretical chemists has been and continues to be improving the accuracy and range of tools that target specific levels in the scale hierarchy. ${ }^{3-7}$ Provided the different levels in the hierarchy are only weakly coupled, such approaches are perfectly adequate for comparison of experiment and theory. Thus ab initio electronic structure calculations are adequate for understanding ultraviolet/visible spectra and classical molecular dynamics can be used to compute infrared spectra. The current challenge is to understand phenomena that are inherently multiscale, implying that two or more levels in the hierarchy are strongly coupled because of which traditional theoretical or experimental tools are not applicable. This is the case with some of the most interesting phenomena in materials science, chemistry and biology. A familiar example is that of electron transfer, where the quantum mechanical electron transfer process is intimately coupled to solvent reorganizations. ${ }^{8,9}$ An overall theoretical framework was provided by Marcus but the actual simulation of a realistic system still remains difficult. Enzyme catalysis provides another example of such a strongly coupled, multiscale process. ${ }^{10}$ The critical event involving the elementary chemical reaction of bond breaking or formation of the substrate molecule takes place locally and on a time scale of femtoseconds. The steps that precede the catalytic reaction, such as the diffusion of the substrate to the active site and the conformational reorganization of the enzyme necessary to lower the activation energy, are long on molecular time scales. The actual reaction rate constants for the catalytic process are of the order of milliseconds. In order to analyse and predict enzymatic catalysis it is therefore necessary to span electronic, atomic and mesoscopic length scales.

This review will focus on recent developments in physical chemistry which either enlarge the spectrum of length and time scales on which we can probe chemical systems, or allow us to integrate information over many different levels of the length/time scale hierarchy.

Our intention is to illustrate how a range of interdisciplinary problems can be addressed using these novel experimental and computational techniques in conjunction with the standard concepts and tools of physical chemistry.

Section 2 provides an overview of two recent techniques that can probe molecular systems at very small spatial and temporal scales, e.g. ultrafast and single-molecule spectroscopies. These two techniques represent fundamentally novel ways of probing the behaviour of chemical systems and have resulted in new conceptual developments to interpret and design experiments in biology, chemistry and physics. Multi-scale computational and theoretical approaches are reviewed in section 3. Sections 4, 5 and 6 illustrate how specific systems and phenomena can be effectively understood using the experimental techniques and multiple time-scale computational approaches outlined in sections 2 and 3. Section 4 contains a discussion of some current areas of interest in liquid state dynamics and solvation. Protein folding and aggregation are regarded as among the most consequential and difficult problems of structural biology and form the subject of section 5 . Section 6 considers phase transition equilibria and kinetics, specifically with reference to nanoscale self-assembly.

\section{Experimental tools for probing length and time scales}

Spectroscopy and scattering techniques are wellestablished tools for probing molecular structure and dynamics. Depending on the wavelength of the incident radiation, these techniques can be used to probe the system at atomic, electronic or nano-scales. Optical microscopes are increasingly used in conjunction with spectroscopy to study dynamical processes at the single molecule level. In this section, we do not attempt to review these state-of-the-art techniques but focus on two recent developments that have dramatically altered our capability to probe the microscopic spatiotemporal correlations in molecular systems.

\subsection{Ultrafast spectroscopy}

$\mathrm{G} \mathrm{N}$ Lewis once suggested that an appropriate unit of time for molecular processes would be the 'jiffy', defined as the time taken by light to travel $1 \mathrm{~cm}$, or 33 picoseconds. Time scales shorter than this may be considered to be 'ultrafast'. Between the late 1960s and early 1980s, the technology developed rapidly to enable the study of chemical reactions in 
the nanosecond, and subsequently, in the picosecond time domain. This period saw the emergence of a microscopic understanding of the dynamics of vibrational energy and phase relaxation and isomerization dynamics. At the same time, it was realized that exploration of phenomena such as solvation dynamics, excited state electron and proton transfer reactions and dynamics at conical intersections required even shorter time resolutions.

The late 1980s saw the birth of femtochemistry with Ahmed Zewail reporting time-resolved experiments to detect the transition states involved in chemical reactions, using what was termed as the 'fastest camera in town'. ${ }^{11,12}$ In his Nobel Prize lecture, Zewail described the crucial components of his camera as: (a) a femtosecond probe laser, determining the shutter speed, (b) a mechanism to record the image of the molecular system, e.g. using massspectrometry, diffraction or spectroscopy (c) a femtosecond pump (initiating laser) which serves to set the zero of time and create a coherent wave packet of molecular states whose motion can be followed in real time. The design and interpretation of femtosecond experiments has required considerable conceptual developments, including exploring the implications of molecular coherence, development of a time-dependent formulation for spectroscopy and appropriate computational tools to interpret femtosecond data. The initial ideas were developed and tested using simple gas-phase reactions, such as dissociation of ICN and alkali halides. In the early experiments, it was possible to observe transition states corresponding to unstable configurations of chemical systems located at the top of potential energy barriers separating reactants and products. Today, the conceptual tools and the experimental techniques of femtochemistry are used to address questions in biophysics, as will be discussed in section 4.

Moreover, it has now become possible to progress from femtosecond spectroscopy to attosecond spectroscopy in order to probe electronic motion. ${ }^{13,14}$

\subsection{Single-molecule spectroscopy}

While ultrafast spectroscopy provides an astonishing array of time scales, its utility is sometimes limited by the absence of spatial resolution. This lacuna has been partly removed by single molecule spectroscopy (SMS) that probes the dynamics of a single molecule in the condensed phase. ${ }^{15-17}$ SMS uses a combination of laser spectroscopy and optical microscopy to achieve both temporal and spatial resolution, and is ideally suited to explore dynamics of protein folding, protein-DNA interaction and enzyme kinetics. The experimental design must be such that concentrations are low enough to ensure that only one molecule is in resonance in the volume probed by the laser. Signal-to-noise ratios must also be high enough that the signal from a single chromophore can be detected despite the large number of solvent molecules and the intrinsic noise of the measurement. With recent advances in the field of microscopy, like confocal illumination and twophoton microscopy, single molecule spectroscopy can completely remove ensemble averaging and therefore allow the underlying distributions to be determined, providing a powerful probe of static heterogeneity due to different nanoenvironments or conformational variations. Time resolved variants of SMS spectroscopy can also address the dynamic heterogeneity associated with the time-dependent variations in the behaviour of a macromolecule, such as a protein. The review by Moerner discusses a number of milestones in SMS, including the possibility of creating single molecule sources of single photons on demand. ${ }^{15}$

Single-molecule spectroscopy has proved to be particularly useful in probing the structure and function of proteins. ${ }^{16,17}$ For example, SMS experiments offer the possibility of directly examining the timedependent folding processes of a single protein molecule. By encapsulating individual protein molecules inside unilamellar protein vesicles tethered to surfaces, the time-dependent folding and unfolding behaviour of adenylate kinase was observed using fluorescence resonance energy transfer (FRET). In the case of enzymatic catalysis, the work of Xie and others demonstrates how static and dynamic heterogeneity can be experimentally studied in the case of redox reaction rates for avoenzymes. ${ }^{17}$ They demonstrate a molecular memory phenomenon, where enzymatic turnover depends on previous turnovers, due to slow conformational fluctuations.

\section{Integrating information over multiple length and time scales}

\subsection{Multiscale computational methodologies}

Computation is now frequently projected as a tool for discovery, complementing the traditional scien- 
tific methods of theory and experiment. ${ }^{18-22}$ This is particularly true for molecular systems in which
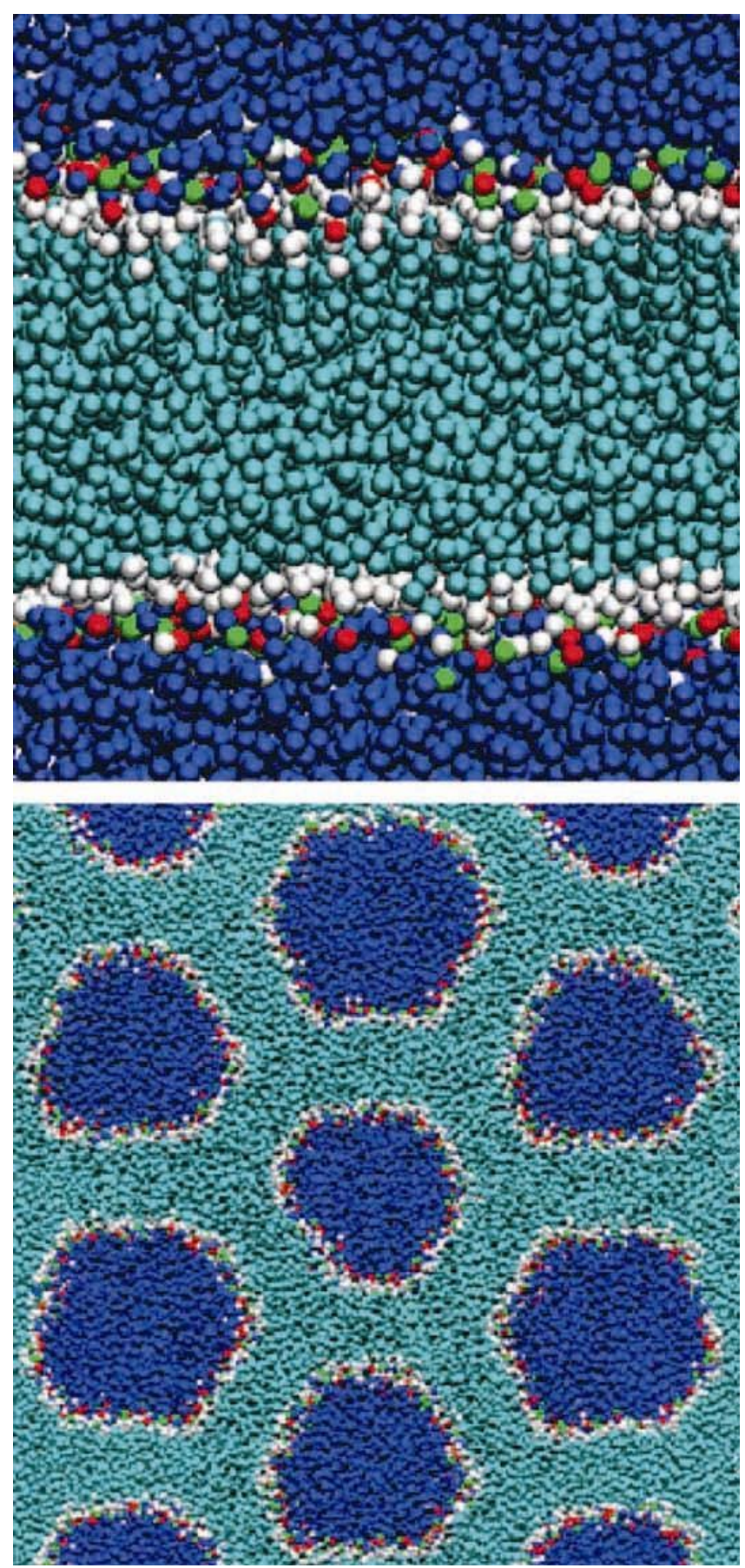

Figure 1. Snapshots of a bilayer formed by a $1: 1 \mathrm{mix}-$ ture of cationic phospholipids stearoyl-decanoylethylphosphatidylcholine (C18:0/C10-EPC) and anionic phospholipid dioleoylphosphatidyl glycerol DOPG at $298 \mathrm{~K}$ (top) and an inverted hexagonal phase of a $1: 1$ mixture of oleoyl-decanoyl-ethylphosphatidylcholine (C18:1/C10-EPC) and DOPG at $333 \mathrm{~K}$ (bottom). The coarse-grained model used to simulate the lipid-water mixtures is discussed in ref. 21. (Reproduced with permission of American Chemical Society). computer simulation techniques play a central role. For such systems, computational techniques are valuable not just for understanding the microscopic details, but also for exploring unexpected structures and emergent properties. As mentioned in the introduction, computational tools for addressing separate length and time scales in the hierarchy are relatively well developed. The current challenge is to develop efficient multiscale simulation methodologies which can integrate information from different levels of the hierarchy, particularly with a view to addressing problems on the nano- or meso-scale. Figures 1 and 2 address two such recent applications to intrinsi-
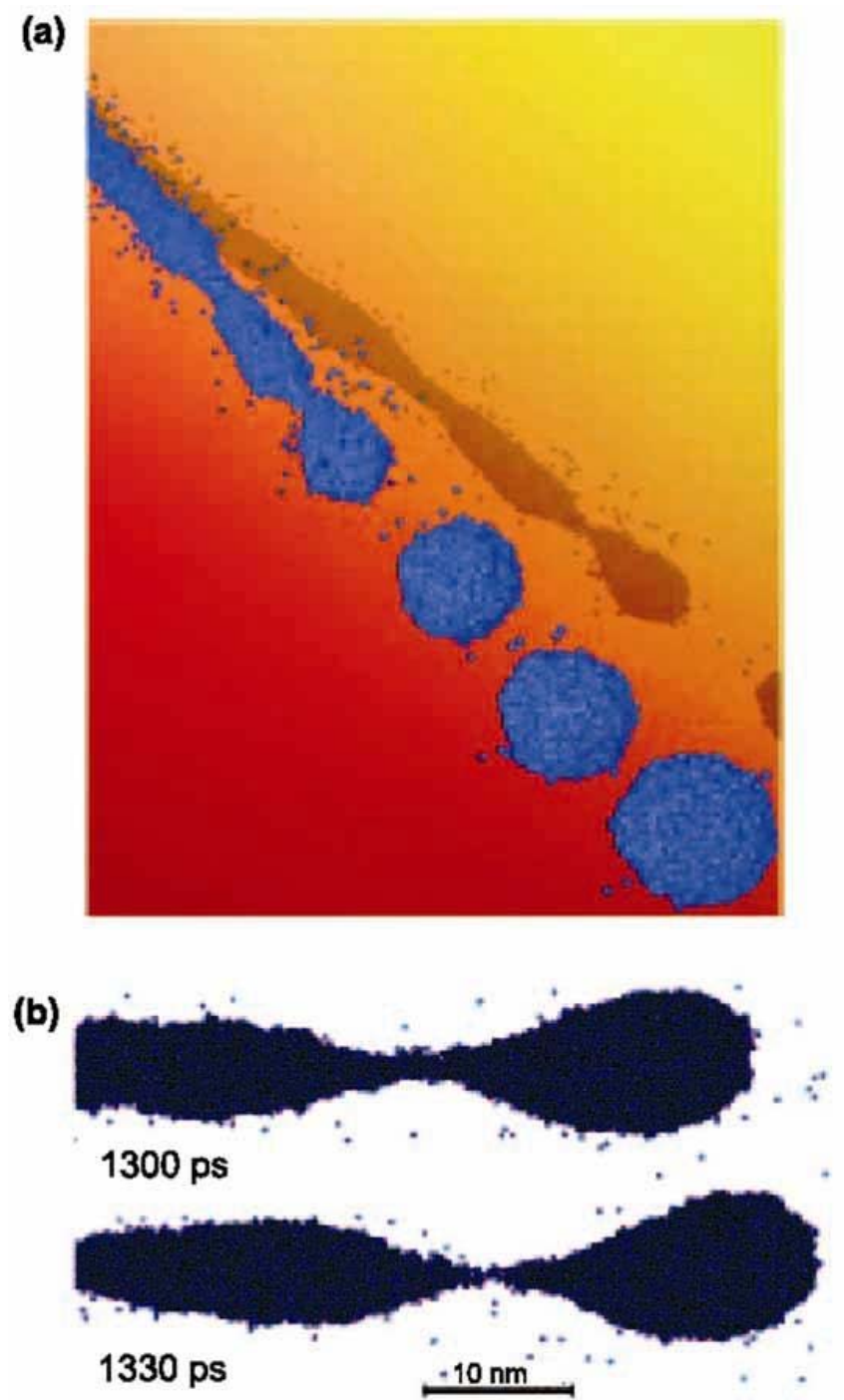

Figure 2. Atomistic configurations obtained with MD simulations of propane nanojets emanating from a $6 \mathrm{~nm}$ diameter nozzle, illustrating the development of instabilities and a double-cone pinch-off configuration (see ref. 18). Each small blue sphere represents a propane molecule. (Copyright 2005 National Academy of Sciences, USA). 
cally multi-scale problems. Figure 1 shows a picture of a lipid bilayer taken from ref. 21. The structure, dynamics and phase transitions of such a bilayer are strongly dependent on hydrophobic interactions, and therefore on the solvent molecules and their interaction with the macromolecular lipids. Some aspects of such a bilayer can, however, be treated at a coarse-grained level that ignores atomistic details; for example, a lipid bilayer can be treated as a twodimensional membrane characterized by macroscopic variables, such as local measures of elasticity. Figure 3 shows the formation of nanojets as propane gas escapes from a nozzle. ${ }^{18}$ Formation and break-up of liquid jets are typically modeled using continuum fluid dynamics but the continuum approximations must be modified when the jet diameter approaches the nanoscale. Atomistic simulations provide a means to suggest as well as test such coarse-graining strategies.

The most tractable version of multiscaling is to use a sequential approach where simulations of relatively small systems at lower levels of the hierarchy

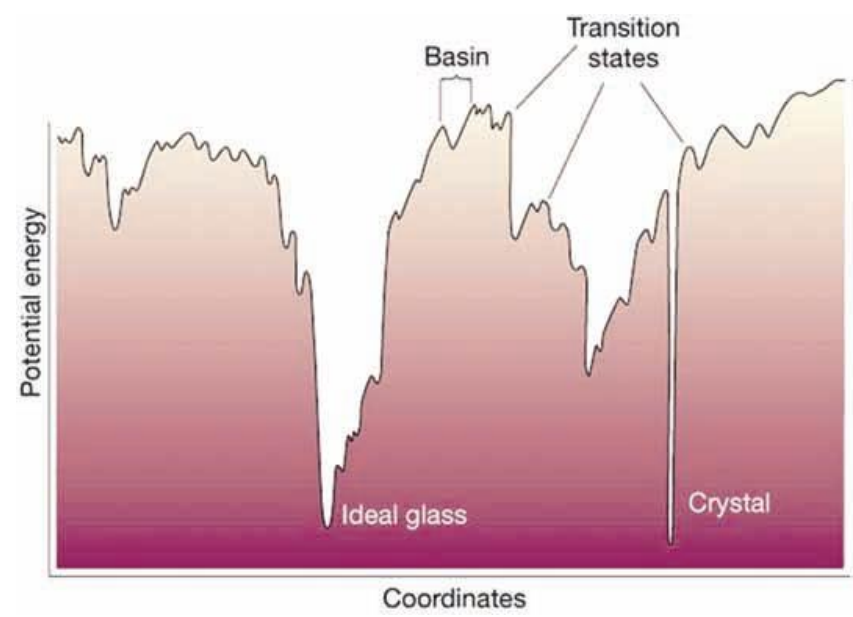

Figure 3. Schematic illustration of the potential energy landscape of the condensed phases of an atomic system, taken from ref. 31. The potential energy will be a function of the position coordinates of all the atoms which is represented by the $x$-axis in this schematic diagram. The ordered low energy state corresponding to the perfect crystal is the global minimum. The ideal glass state is an amorphous, packing minimum with energy very close to that of the crystal. In the liquid phase, the system makes frequent transitions between basins of high energy local minima. Crystallisation requires that the system find its way from the manifold of high energy minima to the basin of the global minimum. Vitrification corresponds to trapping of the system in a metastable minimum. Note the similarities with the free energy landscape for protein folding shown in the next figure. (Figure reproduced with permission of Nature Publishing Group). are used to generate input for higher levels in the hierarchy. ${ }^{19,20}$ A familiar example is the use of electronic structure calculations to fit the parametric functional forms used to represent interatomic interactions which can then serve as input for atomistic simulations. ${ }^{23}$ This level of coarse-graining can be rigorously justified by the large difference in electronic and atomic time scales underlying the BornOppenheimer approximation. Moving from the atomic to the nano-scale requires some thought regarding the appropriate type of information to be derived from the atomistic simulations in order to be used as input for the mesoscale simulations. One approach is to construct effective potentials acting on the most important degrees of freedom, while averaging or integrating over the other atomistic degrees of freedom, such as those of the solvent molecules. ${ }^{19-21,24-26}$ Unlike in the case of interatomic potentials, these effective potentials depend on the thermodynamic state point. Rigorous approaches to the coarse-graining attempt to preserve the partition function but when this is not possible, the coarsegraining strategy is designed to ensure that certain key thermodynamic quantities are preserved. The snapshot of a lipid bilayer shown in figure 2 was taken from a simulation using such a coarse-grained potential. When studying mechanical properties of materials, atomistic simulations may be used to provide information on energies and structures associated with crystalline defects or dislocations.

Unlike sequential methods, concurrent approaches to multiscaling attempt to simultaneously simulate the system at different levels of the hierarchy. Such methods are, in principle, more powerful than sequential models because they make no assumptions about coarse-graining models and can be applied when different levels of the hierarchy are strongly coupled. An example of a highly successful multiscale approach is ab initio or Car-Parrinello molecular dynamics that is appropriate when electronic structure and atomic scale motions are strongly coupled, as in chemical reactions or in certain categories of phase transitions in which the nature of chemical bonding is altered. ${ }^{5,27}$ Interesting efforts are currently being made to formulate concurrent simulation methods that will link the electronic, atomic and meso/macroscale by combining $a b$ initio methods, classical molecular dynamics and finite element methods, respectively. Examples of such approaches to study mechanical properties of materials, such as plasticity and fatigue, are given in refs $28-30$. 


\subsection{Sampling energy landscapes}

The energy landscape picture provides a convenient framework for understanding phase transition thermodynamics and kinetics in complex chemical systems. ${ }^{31,32}$ Potential energy landscapes focus on the configurational energy as a function of the $3 N$ dimensional position vector of an interacting collection of $N$ atoms. Assuming that quantum effects are negligible on the atomic scale at temperatures of interest, the potential energy surface contains all the information necessary to understand the collective properties. Energy landscape analyses attempt to characterize the significant topographic features of the potential energy landscape, such as number, location and energies of minima and saddle points. The sampling of the different regions of the energy landscape by a system in a given ensemble can be simulated by generating a deterministic or a stochastic trajectory, using molecular dynamics or Monte Carlo simulations. An alternative is to focus on the free energy of the system as a function of collective variables or order parameters. While the potential energy landscape picture emerges naturally from a molecular simulation perspective, free energy land scapes emerge naturally from a density functional approach. ${ }^{4}$

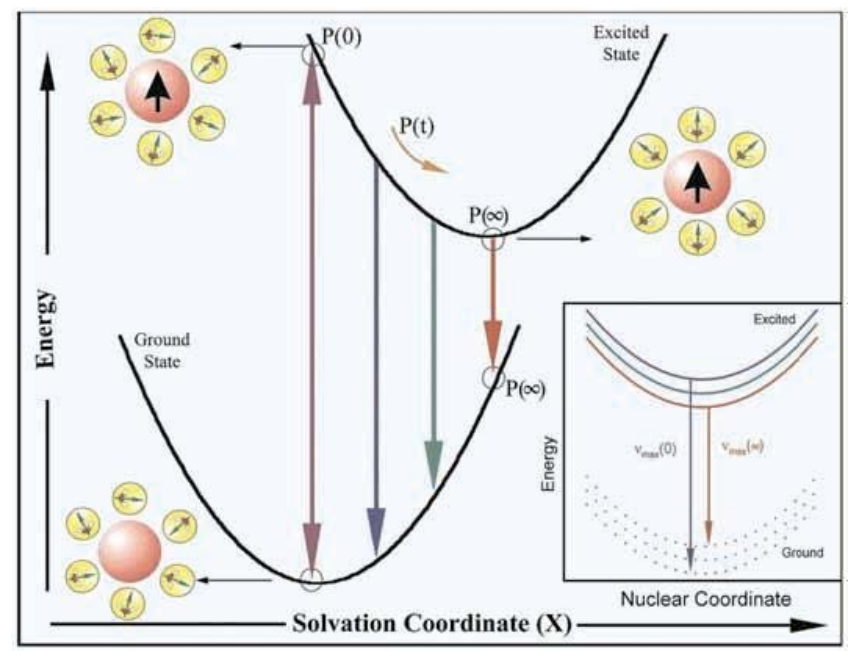

Figure 4. Schematic illustration of the potential energy surfaces involved in solvation dynamics showing the water orientational motions along the solvation coordinate together with instantaneous polarization $P$ (see ref. 52). In the inset we show the change in the potential energy along the intramolecular nuclear coordinate. As solvation proceeds the energy of the solute comes down giving rise to a red shift in the fluorescence spectrum. Note the instantaneous $P$, e.g. $P(¥)$, on the two connected potentials (see refs 51,52 ).
The potential energy landscape paradigm was originally formulated in the context of supercooled liquids (see figure 4). In the solid phase, the system is located in the basin of the global minimum while in the liquid phase, it can make rapid transitions between a manifold of metastable minima. The properties of the local minima and saddles of this energy landscape determine the thermodynamic and kinetic properties of the phases. For supercooled liquids, the energy landscape picture has yielded important insights into phenomenon such as melting, supercooling and the glass transition.

The energy landscape view of liquids can be extended very successfully to understand orderdisorder transitions in complex fluids, such as the isotropic-nematic transition in liquids ${ }^{33}$ and folding of a solvated polypeptide chain into the native protein structure. ${ }^{34}$ In the case of protein folding, it is more natural to think in terms of a free energy landscape by averaging over the solvent degrees of freedom, rather than the potential energy landscape. The native, folded configuration of the protein can be regarded as lying in the global minimum of the system while the unfolded and partially folded states lie in metastable free energy minimum. This energy landscape view of protein folding is discussed in greater detail in section 5 . The complexity of a chemical system can be defined in terms of the structure of the energy landscape, including the multiplicity of minima, the structure of the basins of minima and their connectivity. The time-scales governing the system dynamics will be associated with bottlenecks in crossing between local minima. To understand the dynamics on a free energy landscape, it is convenient to start with the simplest version of the problem i.e. to view the chemical reaction between two species A and B as a transition between two free energy minima. The computational approach to determine the reaction rate for such a process from a molecular dynamics simulation is based on the transition state theory and was formulated by Bennett and Chandler in the late 1960s. ${ }^{3}$

More recent algorithms to bridge time scales in complex systems can be viewed as strategies to construct transition paths between minima separated by free energy barriers of either enthalpic or entropic origin. If the key collective variables likely to influence the dynamics are known, then methods such as umbrella sampling or parallel tempering can be used to construct the free energy surface. For example, in the case of solid-liquid phase transitions, the degree of crystallinity is clearly a suitable order parameter. 
Frequently, however, it is not possible to form an $a$ priori judgement of the critical collective variables in a complex system. The current interest centres on approaches to address precisely this class of problems, such as transition path sampling, ${ }^{35}$ hyperdynamics ${ }^{36}$ and metadynamics. ${ }^{37}$ As the number of accessible minima in the free energy landscape grows, corresponding to a multiplicity of possible reactants and products connected by elementary reactions, it is often necessary to adopt a multiscale kinetic Monte Carlo (KMC) approach. For example, for surface growth phenomena, KMC simulations combining kinetic energy barrier parameters for relevant elemental processes from ab initio simulations can be used to predict spatiotemporal patterns associated with surface growth. ${ }^{38}$ In the asymptotic limit of extreme complexity found in many chemical, biological or genetic networks, sophisticated algorithms may be required to merely deduce the relevant species and the reaction mechanism connecting them. ${ }^{39}$

\section{Liquids and solvation}

Much of chemistry takes place in the liquid state with the solvent playing a critical role in determining the overall thermodynamics and kinetics of various processes, including those associated with protein folding and nanoscale self-assembly discussed later. The dynamics of a liquid spans a huge range of time scales - from ultrafast molecular level reorganisations to very slow dynamics associated with supercooling and the glass transition. Theories of the liquid state are among the more traditional domains of physical chemistry, but many of the techniques and ideas are currently being employed to address problems in complex fluids and soft matter. In this section, we consider the behaviour of a specific and ubiquitous solvent (water), as well as some general features of solvation dynamics and the glass transition.

Since water is the most common solvent for chemical and biological systems, understanding bulk water and its behaviour as a solvent is of considerable interest. ${ }^{40,41}$ Water has long been known to be an anomalous liquid in terms of its dielectric, thermodynamic and kinetic properties. Computer simulations for water pose a challenge in that the significant contribution of both dispersion and many-body polarizability contributions to the intermolecular interactions limit the accuracy of both classical and
Car-Parrinello simulations. The light hydrogen atoms imply that quantum simulation methods are required for accuracy. ${ }^{42,43}$ Despite these limitations, classical Monte Carlo and molecular dynamics simulations, in conjunction with experiments, have resulted in uncovering a number of unexpected features in the phase diagram of water, including new metastable phases of ice and distinct polyamorphic (or glassy) forms. ${ }^{44,45}$ An unexpected finding has been that the anomalous properties of water (such as the rise in density on isobaric heating) are not uniquely associated with the tetrahedral, liquid-state network of water but may be seen in liquids with isotropic interparticle interactions. ${ }^{46,47}$

The hydrophobic interaction, most commonly manifested as the segregation of oil and water, was identified by Kauzmann in 1959 as the driving force in the collapse of a protein to its folded structure. ${ }^{48}$ A molecular theory of hydrophobicity is essential for understanding nanoscale organisation of biological structures and for developing appropriate simulation methodologies at both atomistic and mesoscopic scales (see figure 2). Recent work shows that there is a strong length scale dependence in hydrophobic interactions. ${ }^{49}$ Small hydrophobic solutes induce some local order in the surrounding water but do not change the number of hydrogen bonds in bulk water significantly. A large hydrophobic interface, however, actually disrupts the hydrogen-bond network of water. The scaling of the free energy of hydration with the size of the hydrophobic solute therefore changes qualitatively at a length scale of approximately $1 \mathrm{~nm}$. In the context of biomolecular interfaces, the molecular picture of hydrophobicity is further complicated by the existence of surface chemical heterogeneity, since the protein surface will typically have both hydrophobic and hydrophilic patches. ${ }^{50}$

The dynamics of the solvation process, as described by the time-dependent response of the solvent to sudden changes in properties of the solute, was one of the first areas of chemistry where time-resolved techniques were successfully applied. Solvation dynamics has been extensively used to obtain important information on a wide variety of systems, from dipolar liquids like water and acetonitrile to complex systems like proteins, DNA molecules, and micelles ${ }^{51-56}$ A schematic illustration of solvation as a dynamical phenomenon probed by time domain laser experiments is shown in figure 4. One can experimentally construct a solvation time correlation 
function (STCF) that measures the progress of solvation. This time scale and also the exponentiality/ non-exponentiality of the STCF serves to characterize the intrinsic dynamics of the complex solutesolvent system. From the perspective of statistical mechanics, one can use linear response theory to describe the solvation time correlation function in terms of an energy-energy time correlation function which is then related to the wavenumber and frequency dependent dielectric function. The latter can be obtained either from simulations or from an analytical theory within certain approximations. The agreement between theory, simulations and experiments has been tested for a diverse range of systems and proven to be very satisfactory. It is generally agreed that solvation dynamics in pure liquids measures dynamics over rather short distances, although long range collective effects also play a role. Bulk dipolar solvents, such as water and acetonitrile, were found to exhibit ultrafast solvation dynamics with sub-100 fs time constants though confinement of the solvents in reverse micelles can lead to a very slow solvation time scale.

Hydration dynamics at biomolecular interfaces shows a number of interesting features which have initiated a large amount of controversy and discussions. ${ }^{41}$ At the protein surface, water molecules are influenced both by the protein and the bulk water. It is now believed that water molecules form a two dimensional network structure around the protein molecule, with an associated modification of water structure up to about 2-4 molecular layers of water. Several recent studies have explored water solvation and orientational dynamics in the major and minor grooves of DNA. ${ }^{56}$ The slow dynamics in the minor groove was interpreted partly as due to nanoconfinement and partly due to interaction with the polar base atoms in the minor groove.

As liquid is supercooled, it can either undergo a nucleation process to form the stable solid or undergo a glass transition. The glass transition is experimentally defined as the temperature at which the viscosity of a liquid reaches $10^{13}$ poise. The key features of the glass transition are the simultaneous appearance of slow non-exponential relaxation and non-Arrhenius temperature dependence. ${ }^{57,58}$ Understanding the nature of the glass transition is an important issue in condensed matter theory and statistical mechanics. Given the technological importance of glass in a variety of contexts (e.g. metallic glass and amorphous silicon), the glass transition has been subject of considerable experimental and theoretical work. A number of different theoretical approaches have been developed to understand the glass transition. Energy landscape approaches have been employed to understand the connection between thermodynamics and dynamics in the stable and metastable supercooled liquid state. ${ }^{59}$ Mode coupling theory can successfully explain the initial growth of relaxation time on approaching the glass transition but is known to falter at still lower temperatures where viscosity becomes larger than approximately 100 poise. $^{60,61}$ An important issue is the dynamic correlation length which characterizes cooperatively rearranging regions (CRR) of the liquid that emerges as the glass transition is approached. Both experiments and theory suggest a size of a few $\mathrm{nm}$ for these systems. It has been suggested that the morphology of these CRRs will show a crossover from being fractal, close to the mode-coupling temperature to compact structures, close to the glass transition. ${ }^{62}$

\section{Protein folding and aggregation}

Understanding the physical principles that govern the folding of an extended polypeptide chain into the characteristic three-dimensional tertiary structure or 'native' state constitutes one of the fundamental problems of natural science. Since Anfinsen's statement of the thermodynamic hypothesis more than half-a-century ago, the protein folding problem has been an area where experiment, theory and computations have interacted very effectively. ${ }^{63-66}$ The thermodynamic hypothesis states that 'sequence dictates structure', i.e. the native state represents the minimum free energy structure for the polypeptide chain in its physiological millieu. Protein folding times under physiological conditions are of the order of nanoseconds to milliseconds. The Levinthal paradox encapsulates the difficulty of reconciling the enormous number of conformational structures with relatively short protein folding time scales. The diversity of folding times and the complexity of the intracellular millieu, however, implies that sequential folding pathways guided solely by thermodynamic stability are likely to exist only in a small category of fast-folding proteins. The challenge of understanding protein folding is complicated by the fact that computer simulations of the energetics and dynamics of a polypeptide chain in an aqueous medium are limited in terms of simulation size, length of time for which the protein dynamics can be 
followed and the accuracy with which the underlying interatomic interactions can be modeled. Despite the complexity of the problem, a combination of experimental techniques (NMR, optical spectroscopies, crystallography), multi-scale and coarsegrained simulations and insights from the statistical mechanics of liquids and polymers have led to the development of an energy landscape paradigm as a conceptual framework for understanding protein folding. Figure 5 shows the free energy landscape of a typical protein, constructed using simulation and NMR data. The free energy is mapped out as a function of two key collective variables: the number of native interactions and the number of residue contacts. The various protein conformations can be seen to occupy different parts of a funnel in the free energy landscape which terminates at the native structure. The unfolded, random coil configurations of the protein occupy the mouth of the channel. Intermediate states formed by hydrophobic collapse and secondary structure formation lie along the funnel and define the folding pathways. The shortest time-scales in protein folding are associated with the

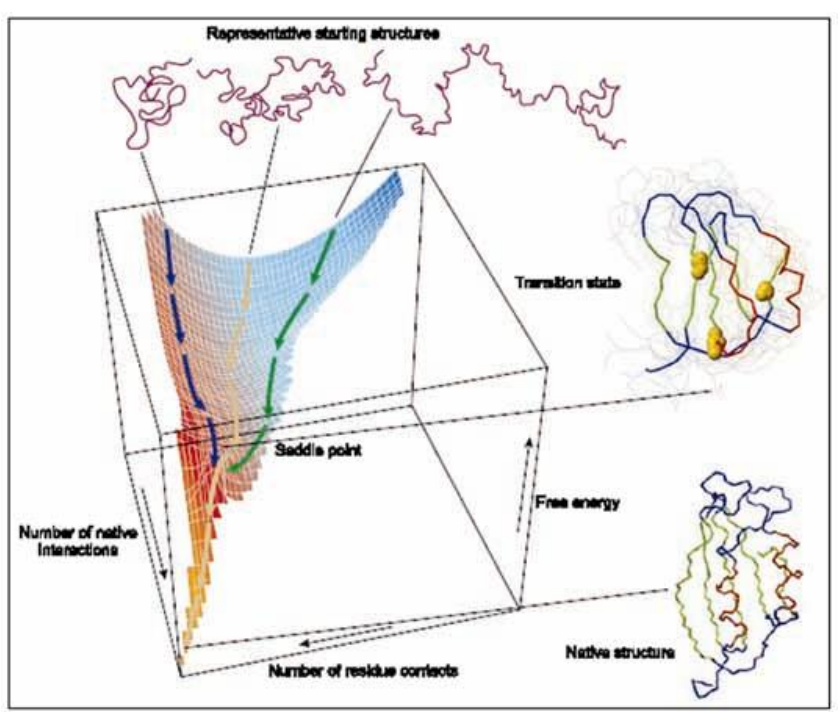

Figure 5. A schematic free energy landscape for protein folding. The surface is derived from a computer simulation of the folding of a highly simplified model of acylphophatase, with constraints derived from experimental data from mutational studies. The yellow spheres in this ensemble represent the three 'key residues' in the structure; when these residues have formed their native-like contacts the overall topology of the native fold is established. The native state lies at the bottom of the free energy funnel and possible folding pathways are shown with arrows (figure reproduced with permission of Nature Publishing Group). formation of secondary structures, such as helices while the long time scales are associated with the formation of tertiary structures involving contacts between amino acid residues well-separated along the primary amino acid sequence. The landscape picture can accommodate multiple pathways and transition state ensembles. When entropic and enthalpic contributions to the free energy compensate, there may be no significant barriers to folding, leading to very fast, 'downhill' folding.

The existence of complex protein folding pathways implies that in the cellular environment, the intermediate structures are available for interaction with other intracellular components. Some of these may be molecular chaperones which assist the folding process whereas others may lead to misfolding. Protein aggregation, specially the formation of fibrillar aggregates, leads to severe pathologies. The nucleation processes which lead to folding versus aggregation are consequently an active area of research. Another active area of research where many of the ideas of protein folding and aggregation can be applied is in biomimetic strategies for creating non-biological polymers that self-assemble to form stable, unique structures in different solvents. ${ }^{67-69}$

\section{Phase transitions, nucleation and self-assembly}

The equilibrium phase diagram of a system summarises the range of thermodynamically stable bulk structures that can be generated from a set of atomic or molecular constituents. The generation of phase diagrams for a range of systems is today possible using a variety of simulation techniques, where the predictive accuracy of the simulations depends largely on the accuracy with which the underlying interactions can be modeled. ${ }^{3}$ The kinetics of phase transformations, in contrast to the thermodynamics, is currently an active area of work. First-order transitions are assumed to form by a nucleation process where an embryonic stable phase forms within the metastable phase, in the absence (homogeneous) or presence (heterogeneous) of surfaces or impurities. Classical nucleation theory assumes that free energy required to create a nucleus of the stable phase in a metastable matrix depends on two competing terms: a surface term that represents the free energy required to form an interface and a volume term that represents the free energy gain proportional to the 

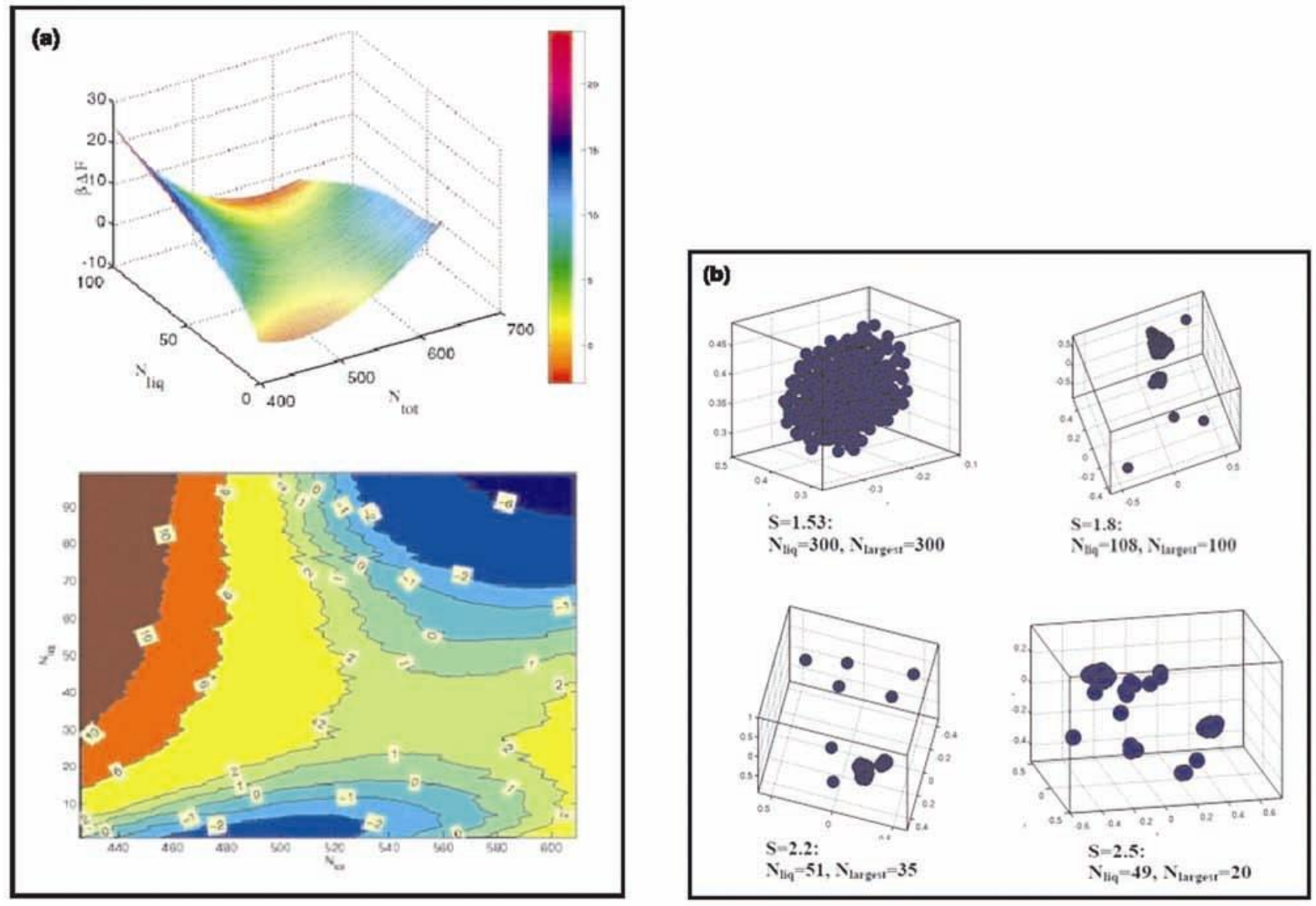

Figure 6. (a) The 3-dimensional free energy surface (upper panel) and corresponding 2-dimensional contour plot (lower panel) computed in grand canonical $(\mu \mathrm{VT})$ ensemble at supersaturation $\approx 2.4$ (near gasliquid spinodal). (b) Snapshots of the system at four different supersaturation $\left(S=P / P_{\mathrm{c}}\right.$, where $P$ is the pressure of the system and $P_{\mathrm{c}}$ is the same at the gas-liquid coexistence.): They show all the liquid-like particles of the system at the top of the barrier. For higher supersaturation, we find multiple large clusters are forming around the critical cluster and growth of the liquid phase becomes spread over the whole system rather than the single 'critical cluster' (see ref. 74).

difference in chemical potential between the stable and metastable phases. ${ }^{57}$ Nuclei greater than a critical size will grow spontaneously. The formation of a nucleus of critical size is thus the rate determining step and takes place on time scales that are very long compared to molecular time scales. Since a complex system can form multiple phases, crystallization from a melt can frequently result in the formation of metastable phases rather than stable phases.

Appropriate simulation methodologies to bridge time scales for nucleation in molecular simulations are currently an active area of work. Computational studies of nucleation have largely focused on the condensation and crystallisation processes in relatively simple one-component systems that allow one to address a number of interesting theoretical possibilities, including the effect of metastable phases on crystallization and condensation in the vicinity of the liquid-gas spinodal. ${ }^{70-74}$ One finds that at large supersaturation (near the spinodal), the mechanism of nucleation undergoes a remarkable change from classical single particle addition to a collective mode, spread over the entire system (figure 6). The critical step is now the coalescence of clusters of intermediate size on a at free energy surface (figure 6) ${ }^{74}$ These studies have interesting implications for nucleation in more complex systems, e.g. crystallization of proteins or obtaining stable polymorphs of pharmaceuticals

Phase transitions under equilibrium and nonequilibrium conditions are of special interest in the context of nanoscale self-assembly of ordered structures. ${ }^{75-78}$ Advances in nanoparticle synthesis have made it possible to synthesize nanoparticles in a large variety of shapes and sizes. These nanoparticles can self-assemble to form a remarkable range of ordered structures (see figure 7 for an illustration of a miniscule sample of possible structures). Under a 

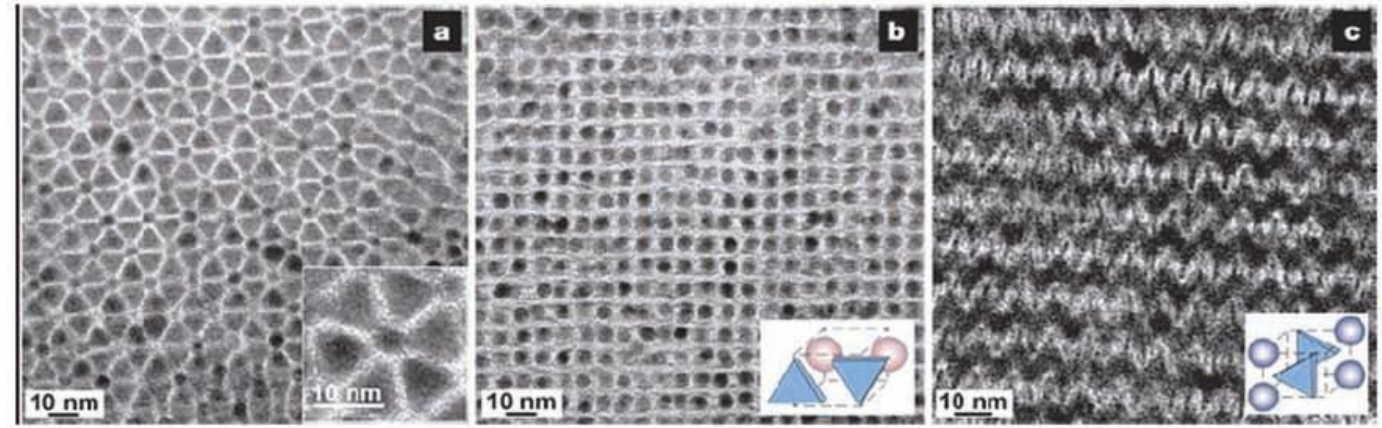

Figure 7. TEM images of self-assembled structures and proposed unit cells of binary superlattices (a) and (b) structure formed from LaF3 triangular nanoplates ( $9.0 \mathrm{~nm}$ side) and $5.0 \mathrm{~nm} \mathrm{Au}$ nanoparticles; (c) structure formed from LaF3 triangular nanoplates and $6.2 \mathrm{~nm}$ $\mathrm{PbSe}$ nanocrystals. Details are available in ref. 76. (Figure reproduced with permission of Nature Publishing Group).

given set of macroscopic conditions which may not necessarily be equilibrium conditions, an ordered self-assembled structure is formed as a consequence of the interplay between the various contributions to interparticle interactions, such as dispersion, shortrange repulsion, electrostatic, bonding and solvophobic interactions. Understanding the organisational principles of self-assembly and developing the appropriate predictive tools is currently a major challenge.

\section{Concluding remarks}

This article attempts to provide an overview of the interdisciplinary array of theoretical, computational and experimental approaches that are currently being developed in order to understand the interplay between multiple length and time scales that determine structure and dynamics in many chemical systems. The choice of illustrative examples has been inevitably biased by our own research interests but we hope that it is sufficient to illustrate that currently available techniques can address a number of interesting problems in the areas of materials science, chemical biology and condensed matter physics. Constraints of space in a volume of this type have meant that we have not been able to review experimental and theoretical research on areas of related interest in India. There are currently several excellent groups working in the area of spectroscopy, scattering, electronic structure methods, statistical mechanics, liquid state theory, atomistic simulations and continuum methods. These groups are typically dispersed over chemistry, physics, chemical engineering and materials science departments. We hope that this article will illustrate that there is considerable scope as well as need for "mul- tidisciplinary' as well as 'multiscale' approaches to understand a wide range of problems.

\section{Acknowledgements}

This work was supported in parts by grants from Department of Science and Technology (DST) and Council of Scientific and Industrial Research (CSIR), India. We thank Sangeeta Saini, Abir Ganguly, Shadrack Jabes and Biman Jana for help in preparing the manuscript.

\section{References}

1. Pople J 2003 Quantum chemical models in Nobel lectures; Chemistry 1996-2000 (ed.) I Grenthe (Singapore: World Publishing Co.)

2. Kohn W 2003 Electronic structure of matter-wave functions and density functionals in Nobel lectures; Chemistry 1996-2000 (ed.) I Grenthe (Singapore: World Publishing Co.)

3. Frenkel D and Smit B 2002 Understanding molecular simulations: from algorithms to applications (San Diego: Academic Press)

4. Hansen J P and Smit B 2006 Theory of simple liquids (San Diego: Academic Press)

5. Martin R M 2004 Electronic structure: basic theory and practical methods (Cambridge University Press)

6. Roy R K, Krishnamurti S, Geerlings $\mathrm{P}$ and Pal S 1998 J. Phys. Chem. A102 3746

7. Mukherjee D and Pal S 1989 Adv. Quant. Chem. 20291

8. Marcus R A 2003 Electron transfer reactions in chemistry: theory and experiment in Nobel lectures; Chemistry 1996-2000 (ed.) B G Malmstorm (Singapore: World Publishing Co.)

9. Marcus R A 1965 J. Chem. Phys. $\mathbf{4 3} 679$

10. Henzler-Wildman K A, Lei M, Thai V, Kerns J S, Karplus M and Kern D 2007 Nature 450913

11. Zewail A H 2000 J. Phys. Chem. A104 5660

12. Asplund M C, Zanni M T and Hochstrasser R M 2000 Proc. Natl. Acad. Sci. 978219 
13. Kling M F and Vrakking M J J 2008 Ann. Rev. Phys. Chem. 59463

14. Mukamel S, Abramavicius D, Yang L J, Zhuang W, Schweigert I V and Voronin D V 2009 Acc. Chem. Res. $\mathbf{4 3} 553$

15. Moerner W E 2002 J. Phys. Chem. B106 910

16. Lu H P, Xun L and Xie X S 1998 Science 2821877

17. Weiss S 1999 Science 2831676

18. Landman U 2005 Proc. Natl. Acad. Sci. 1026671

19. Brandt A, Bernholc J and Binder K (eds) 2001 Multiscale computational methods in chemistry and physics (IOS Press)

20. Lu G and Kaxiras E An overview of multiscale simulations of materials; arXiv: cond-mat/0401073

21. McCullagh M, Prytkova T, Tonzani S, Winter N D and Schatz G C 2008 J. Phys. Chem. B112 10388

22. Murtola T, Bunker A, Vattulainen I, Deserno M and Kattunen M 2009 Phys. Chem. Chem. Phys. 111869

23. Stone A J 1997 Theory of intermolecular forces (Oxford University Press)

24. Marrink S J, Risselada H J, Ye.mov S, Tieleman D P and de Vries A H 2007 J. Phys. Chem. B111 7812

25. Ballau. $\mathrm{M}$ and Likos C N 2004 Angew. Chem. Int. Ed. 432998

26. Louis A A, Bolhuis P G, Hansen J P and Meijer E J 2000 Phys. Rev. Lett. 852522

27. Car R and Parinello M 1985 Phys. Rev. Lett. 552471

28. Farkas D, Willemann M and Hyde B 2005 Phys. Rev. Lett. 94165502

29. Abraham F F and Broughton J Q 1998 Comp. Mat. Sci. 101

30. Li J, Van Vliet K J, Zhu T, Yip S and Suresh S 2002 Nature 418307

31. Debenedetti P G and Stillinger F H 2001 Nature 410 259

32. Wales D J 2003 Energy landscapes: with applications to clusters, biomolecules and glasses (Cambridge University Press)

33. Chakrabarti D and Bagchi B 2006 Proc. Natl. Acad. Sci. 1037217

34. Onuchic J N, Luthey-Schulten $Z$ and Wolynes P G 1987 Ann. Rev. Phys. Chem. 48545

35. Dellago C, Bolhuis P G and Geissler P L $2002 A d v$. Chem. Phys. 1231

36. Voter A F 1997 Phys. Rev. Lett. 783908

37. Barducci A, Bussi G and Parrinello M 2008 Phys. Rev. Lett. 100020603

38. Voter A F, Montalenti F and Germann T C 2002 Ann. Rev. Mat. Res. 32321

39. Ross J 2008 J. Phys. Chem. A112 2134

40. Franks F (ed.) 1972 Water: a comprehensive treatise (Plenum Press)

41. Bellissent-Funel M C (ed.) 1999 Hydration processes in biology (IOS Press)

42. Tuckerman M E, Marx D, Klein M L and Parrinello M 1997 Science 275817

43. Chakravarty C 1997 Int. Rev. Phys. Chem. 16421

44. Lobban C, Finney J L and Kuhs W F 1998 Nature 391268
45. Mishima O and Stanley H E 1998 Nature 396329

46. Sadr-Lahijany M R, Scala A, Buldyrev S V and Stanley H E 1998 Phys. Rev. Lett. 814895

47. Sharma R, Chakraborty S N and Chakravarty C 2006 J. Chem. Phys. 125204501

48. Kauzmann W 1959 Adv. Prot. Chem. 141

49. Chandler D 2005 Nature 437640

50. Giovambattista N, Lopez C F, Rossky P J and Debenedetti P G 2008 Proc. Natl. Acad. Sci. USA 105 2274

51. Nandi N and Bagchi B 1997 J. Phys. Chem. B101 10954

52. Pal S K, Peon J, Bagchi B and Zewail A 2002 J. Phys. Chem. B106 12376

53. Bagchi B 2005 Chem. Rev. (Review) 1053197

54. Bhattacharyya K 2008 Chem. Commun. 252848

55. Chandra A, Tuckerman M E and Marx D 2007 Phys. Rev. Lett. 99145901

56. Pal S, Maiti P K, Bagchi B and Hynes J T 2006 J. Phys. Chem. B110 26396

57. Debenedetti P G 1996 Metastable liquids: concepts and principles (Princeton: Princeton University Press)

58. Hecksher T, Nielsen A I, Olsen N B and Dyre J C 2008 Nat. Phys. 4737

59. Sastry S 2001 Nature 409164

60. Samanta A, Ali S M and Ghosh S K 2001 Phys. Rev. Lett. 87245901

61. Das S P 2004 Rev. Mod. Phys. 76785

62. Jacob D Stevenson, Jorg Schmalian and Peter G Wolynes 2006 Nat. Phys. 2268

63. An.nsen C B 1973 Science $\mathbf{1 8 1} 223$

64. Dobson C M 2003 Nature 426884

65. Lazaridis T and Karplus M 2003 Biophys. Chem. 100 367

66. Liu F, Du D, Fuller A A, Davoren J E, Wipf P, Kelly J W and Gruebele M 2008 Proc. Natl. Acad. Sci. 105 2369

67. Khakshoor O and Nowick J S 2008 Curr. Opin. Struct. Biol. 12722

68. Robinson J A 2008 Acc. Chem. Res. 411278

69. Prince R B, Barnes S A and Moore J S $2000 \mathrm{~J}$. Am. Chem. Soc. 1222758

70. Auer S and Frenkel D 2004 Annu. Rev. Phys. Chem. $\mathbf{5 5} 333$

71. tenWolde P R and Frenkel D 1997 Science 2771975

72. Ghiringhelli L M, Valeriani C, Meijer E J and Frenkel D 2007 Phys. Rev. Lett. 99055702

73. Bhimalapuram P, Chakrabarty S and Bagchi B 2007 Phys. Rev. Lett. 98206104

74. Whitesides G M and Grzybowski B 2002 Science 295 2418

75. Shevchenko E V, Talapin D V, Kotov N A, O'Brien S and Murray C B 2006 Nature 43955

76. Min Y, Akbulut M, Kristiansen K, Golan Y and Israelachvili J 2008 Nat. Mat. 7527

77. Glotzer S C and Solomon M J 2007 Nat. Mat. 6557

78. Attinger E S and Koumoutsakos P 2004 Multiscale modeling and simulation (Springer) 